\title{
LA LUTTE CONTRE LE RAT MUSQUÉ A L'AIDE D'APPATS EMPOISONNÉS AUX ANTICOAGULANTS
}

\author{
par M. GIBAN \\ (Laboratoire des Petits Vertébrés) \\ (Institut National de la Recherche Agronomique)
}

Par décision en date du 24 juin 1969, le Ministre de l'Agriculture a autorisé, à titre provisoire, la vente de deux spécialités qui sont destinées à préparer des appâts empoisonnés pour détruire le Rat musqué.

La première, dénommée * Ratmustox * (autorisation de vente B 9 016) (1) se présente sous la forme d'un appât prêt à l'emploi et contient $0,07 \mathrm{p}$. cent de coumaféne. La deuxième le * Caid. (autorisation de vente B 1386 ) (2) est un concentrat huileux de chlorophacinone titrant à $0,25 \mathrm{p}$. cent de matière active. Elle était déjà homologuée pour la préparation d'appâts contre les Rats (Surmulot et Rat noir) ainsi que contre le Campagnol des champs.

Une troisième spécialité, le "Quick concentré» (3) qui est aussi un concentrat huileux à 0,25 p. cent de chlorophacinone, a bénéficié de la même extension (autorisation de vente B 7 107).

Dans le même temps, le Ministre de l'Agriculture de Belgique a autorisé l'emploi d'appâts au chlorophacinone, mais en le réservant au personnel de l'organisation officielle de lutte contre le Rat musqué qui opère dans tout le pays

La présente note a pour objet de faire le point de nos connaissances en tenant compte des ètudes faites ces dernières années tant en France qu'à

(1) Commercialisée par la société Trégui, 148, rue du Faubourg de Bourgogne, 45 -Saintlean-de-Braye.

(2) Commercialisée par la société Lipha, 115, avenue Lacassagne, 69 -Lyon $3^{e}$.

(3) Commercialisée par la société Rhône-Poulenc, 22, avenue Montaigne, 75 -Paris $8^{\circ}$. 
l'étranger, ainsi que des conclusions des diverses réunions techniques, nationales ou internationales, qui se sont tenues en 1969, notamment de celle qui eut lieu à Wageningen (Pays-Bas) sous l'égıde de l'Organisation européenne et méditerranéenne pour la protection des plantes (O.E.M.P.P.).

\section{LES ANTICOAGULANTS A EMPLOYER}

Le chlorophacinone et le coumafène sont les deux seuls raticides anticoagulants pour lesquels nous possedons des données précises concernant leur action sur le Rat musqué, grâce tant à des essais de laboratoire qu'à des expérimentations réalisées sur le terrain. II apparait que ce Rongeur possède à l'égard de ces deux toxiques une sensibilité moindre que celle du Surmulot, mais assez comparable à celle du Rat noir.

D'autres anticoagulants peuvent-ils être employés? Cela ne parait pas impossible à priori. Mais les études qui permettraient de l'affirmer font défaut. II convient donc pour l'instant d'en rester aux deux seuls cités, l'emploi de tout autre ne pouvant être envisagé qu'à titre expérimental.

Les conceptions actuellement préconisées pour la préparation des appâts : $0,070 \mathrm{p}$. cent de coumafène et $0,015 \mathrm{p}$. cent de chlorophacinone, sont celles qui ont donné suffisamment satisfaction dans les essais sur le terrain pour que l'emploi de ces deux substances soit autorisé a titre provisoire. Des ètudes se poursuivent à l'heure actuelle pour savoir si ces concentrations sont les plus appropriées, s'il est possible de les abaisser, ou s'il faut, au contraire, les augmenter.

\section{LES APPATS}

Un appât a été très largement employé dans les essais de terrain et dans les premières applications pratiques : c'est la carotte fraiche, coupée en rondelles ou en cossettes (au coupe racine). Le Rat musque la consomme très volontiers et marque pour elle une appétence nette. La pomme parait posséder des qualités analogues, mais à un degré moindre.

Le rat musqué est en réalité un herbivore très polyphage, mais beaucoup de plantes ne sont mangées que de façon occasionnelle ou seulement à la suite d'une accoutumance. La betterave a été utilisée dans certaines destructions expérimentales, seule ou en mélange avec de la carotte. If est probable qu'elle réussit là où le Rat musqué peut en trouver dans les champs voisins.

Il en est de même pour les graines de céréales. Ainsi le Rat musqué consomme volontiers les grains de maïs là où cette plante est cultivée. On peut se demander si la raison des maigres résultats enregistrés avec divers appâts "secs" (grains, agglomérés, biscuits de composition diverse, etc.) ne réside pas essentiellement dans le manque d'habitude de consommer une telle nourriture. Quoiqu'il en soit, il faut noter que l'emploi de grains empoisonnés aux anticoagulants pour la lutte contre le Rat musqué n'est pas actuellement autorisé. Les raisons de cette interdiction sont l'incertitude de leur efficacité et le risque d'accident trop grand à l'égard des oiseaux et autres animaux consommant des grains.

Quant aux légumes deshydrates, les observations faites, en petit nombre il est vrai, montrent que leur acceptation est fort variable. Dans certaines expériences réalisées sur des Rats musqués en cage, des carottes déshydratées étaient mal acceptées. 


\section{PREPARATION DES APPATS AVEC UN CONCENTRAT HUILEUX}

Les observations faites montrent que, lorsque les rondelles de carottes (ou les morceaux de pomme) sont relativement gros, les Rats musqués ont tendance à les emporter dans leur terrier au lieu de les consommer sur place. Le risque d'accident sur d'autres animaux consommant les restes d'appát, se trouve ainsi diminué. En outre, de grosses rondelles se dessèchent ou moisissent moins rapidement que des fines rondelles ou des cossettes. A la vérité on peut se demander si ces appâts transportés sont toujours bien consommés. Les observations sont à poursuivre sur ce point.

Il est donc préférable, pour des raisons de sécuritè et d'efficacité, dutiliser de grosses rondelles (2 cm d'épaisseur environ) chaque fois que la quantité d'appâts nécessaire n'oblige pas à une préparation plus mécanisée des carottes coupées (par passage dans un coupe-racine par exemple). Dans ce cas, la méthode d'empoisonnement décrite par $R$. MOENS parait la meilleure.

Cette préparation est la suivante:
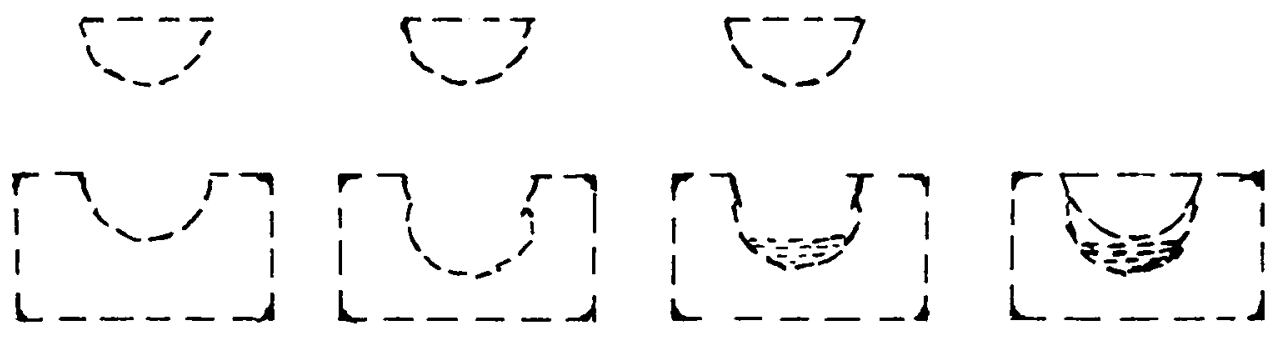

1

2

3

4

1. A l'aide d'un instrument de cuisine appelé moule à pomme de terre noisette, on enlève à chaque rondelle une demi-sphère qui est conservée.

2. La cavité ainsi creusée est approfondie avec le même instrument.

3. On verse dans le fond de la cavité $0,5 \mathrm{cl}$ de concentrat huileux à l'aide d'une pissette en matière plastique.

4. On rebouche la cavité avec la demisphère enlevée en début d'opération

Quelle que soit la forme et la grosseur des carottes coupées, on verse progressivement le concentrat huileux tout en brassant la masse. La dose préconisée $(0,015$ pour cent) conduit à verser 6 litres de concentrat sur $100 \mathrm{~kg}$ de carottes coupées (soit 0,6 । sur $10 \mathrm{~kg}$ ou encore $60 \mathrm{cl}$ sur $1 \mathrm{~kg}$ ). C'est avec beaucoup de soin que le mélange doit être fait et poursuivi jusqu'au moment où tous les morceaux sont enduits d'une pellicule huileuse. L'emploi d'un mélangeur, tel qu'on en utilise pour le traitement des semences par exemple, apparait des plus utiles dans le cas ou de grosses quantités d'appât sont à préparer. Une augmentation de la quantité du concentrat est parfaitement inutile, car l'excédent ne serait pas retenu par les carottes coupées. 


\section{MISE EN PLACE DES APPATS}

Pour la mise en place des appâts empoisonnés, trois questions se posent :

- où disposer les appâts?

- quelle quantité déposer?

- faut-il renouveler les appâts?

Le comportement alimentaire du Rat musqué, c'est-à-dire la façon dont il recherche, trouve et choisit sa nourriture, nous est à peu près inconnu. Certaines observations suggèrent par exemple que le phénomène de néophobie, bien connu chez le Surmulot et le Rat noir, se manifesterait aussi chez le Rat musqué; mais d'autres faits montrent le contraire. Par suite, les règles de mise en place des appâts empoisonnés sont empiriques et il convient de noter soigneusement toutes les observations précises pouvant conduire à une meilleure compréhension du comportement de cet animal.

En règle générale, on placera donc les appâts empoisonnés sur les pistes des Rats musqués repérées le long des berges et dans la végètation aquatique, sur les * salles à manger* (ces petites plateformes où les Rongeurs tirent leur nourriture pour la consommer), dans les terriers ouverts, en hiver sous les huttes, etc.

Aux Etats-Unis, plus précisément en Californie, on a utilisé sur les lacs de retenue d'eau des radeaux, ayant $1 \mathrm{~m} 2$ de surface environ, pour y déposer des appâts empoisonnés. Ceux-ci sont abrités sous une caissette entaillèe de quelques passages pour les Rats musqués. Nous étudions à l'heure actuelle ce procédé qui a l'avantage d'offrir une grande sécurité car peu d'animaux sont susceptibles d'aller chercher les appâts empoisonnès ainsi présentés. Aux premiers résultats, il apparait qu'une période d'accoutumance soit nécessaire et que le procédé ne réussisse guère à l'automne car à cette saison, les Rats musqués utilisent souvent les radeaux pour y construire une fiutte. Les essais se poursuivent néanmoins à d'autres époques.

Il est difficile de répondre à la deuxième question, car si on sait qu'un Rat musqué peut manger de 200 à $300 \mathrm{~g}$ de carotte suivant sa taille, ce n'est pas en une fois qu'il le fait. De plus, il n'est pas possible d'évaluer de façon précise le nombre de Rats musqués qui fréquentent une piste, une coulée, une hutte, etc. Le domaine d'un Rat musqué est relativement vaste et il y a de fortes chances qu'en plaçant les appâts de la façon empirique qui vient d'être indiquée, plusieurs points dempoisonnement se trouveront dans le domaine de chaque animal. Enfin, l'épandage trop généreux des appâts empoisonnés, aura pour conséquence de laisser sur place une quantité importante de restes qui entraineront et aggraveront le risque d'accident sur autres animaux. Aussi, il est conseillé de déposer en moyenne une dizaine de grosses rondelles (que les Rats musqués ont tendance à emporter), ou bien $500 \mathrm{~g}$ de carottes coupées plus finement. Ces quantités seront augmentées aux endroits fréquentés manifestement par un nombre important d'animaux.

La règle fondamentale d'emploi des appâts empoisonnés aux anticoagulants pour la lutte contre le Surmulot et le Rat noir est de renouveler les appâts jusqu'à l'arrêt de la consommation. Cette règle reste vraie pour le Rat musqué parce qu'au moins pour une partie des animaux, il est nécessaire que ceux-ci ingèrent plusieurs fois du toxique. En outre, on n'est jamais certain 
que la quantité d'appâts déposée à un endroit est suffisante pour le nombre de Rats musqués qui le fréquentent.

Une différence existe entre le coumafène et le chlorophacinone: en laboratoire quand ia consommation d'appât empoisonné est limitée à 1 jour, le nombre de Rats musqués qui meurent des suites de cette seule consommation est plus élevé avec des appâts à $0,015 \mathrm{p}$. cent de chlorophacinone quavec d'autres à $0,075 \mathrm{p}$. cent de coumafène par exemple. Mais quand il y a consommation des appâts pendant trois jours consécutifs non seulement cette différence disparait, mais il apparait possible d'abaisser, semble-t-il, la concentration de l'un et l'autre.

Comme il est difficile dans le cas du Rat musqué de songer à un renouvellement journalier des appâts consommés, l'un des buts importants des expérimentations en cours, est de savoir si on ne peut pas obtenir un résultat suffisant, acceptable dans la pratique, par un traitement comprenant un premier épandage, complété par un deuxième deux ou trois jours après. De toute façon, un "entretien" ulterieur est indispersable.

\section{EPOQUE DES TRAITEMENTS}

Deux époques de l'année sont certainement à déconseiller pour la réaiisation d'un empoisonnement des Rats musqués : les entrées de la période de reproduction (février-mars en moyenne) et de celle de l'hivernage (fin septembre et octobre). En effet à ces deux moments, il se produit d'importants mouvements de Rats musqués, qualifiés couramment de migrations. Il est donc préférable d'attendre, pour empoisonner, que les populations soient stabilisées.

On peut se demander si la période de pleine végétation est bien favorable. La nourriture habituelle des Rats musqués étant abondante, les appâts offerts sont-ils vraiment capables de la concurrencer? L'objection n'est pas obligatoirement valable; l'exemple du Surmulot et du Rat noir en fournit la preuve. Si on examine l'ensemble des essais qui ont été réalisés à ce iour, il en ressort que les résultats obtenus ont été moins régulièrement satisfaisants au printemps qu'à l'automne. Mais notre façon d'apprécier les résultats sur le terrain est encore bien grossière. D'autre part, les essais réalisés au printemps sont en nombre plus faible que ceux effectués en automne et hiver.

En définitive, il semble préférable de procéder aux empoisonnements dans la periode qui va d'octobre à janvier, bien que le gel et les variations de niveau d'eau puissent souvent constituer une gêne sérieuse au déroulement des opérations. Mais rien encore ne permet de déconseiller la méthode en période de végétation. Il est par suite particulièrement utile d'expérimenter pour être bien renseigné à ce propos car le début de la période de végétation coincide avec le commencement de la pleine activité de reproduction et il serait intéressant d'intervenir efficacement à ce moment-là.

\section{NECESSITE D'UNE LUTTE COORDONNEE ET GENERALISEE}

Aucune opération de destruction du Rat musqué ne peut être menée à trop petite échelle si on désire un effet non temporaire. En effet les vides créés sont comblés à plus ou moins brève échéance par des individus venus d'à-côté. Ceci est tout à fait général, que le moyen de destruction soit le poison ou le piège.

Ce phénomène se produit même aux périodes où les populations sont 
établies au lieu de reproduction ou d'hivernage. Sa probabilité est encore plus grande aux époques dites de migration.

On ignore à l'heure actuelle de quelles distances peuvent venir ces immigrants Est-ce de quelques centaines de mètres ou de plusieurs kilometres que proviennent ces individus? C'est pour le savoir que les techniques de marquage sont ètudièes à l'heure actuelle.

Une réponse indirecte à cette question a été donnée par les premières expériences d'empoisonnement menées en Belgique sur de grandes surfaces. A la fin de l'année 1968, près de 2000 ha de polders de la région des "Moeren* en Flandre belge ont eté traités aux appâts empoisonnès en deux fois : un traitement principal effectué du 10 septembre au 12 novembre et un traitement complémentaire sitôt apres. II y eut une disparition quasi totale du Rat musqué, dont les réinfestations ont été contrôlées par la pose permanente de nasses dans les canaux, les fossés, etc. La persistance de l'effet obtenu de cette façon était encore totale à la fin de l'année 1969.

Ces expériences ont èté reprises en France et en Belgique dans la deuxième moitié de l'année 1969, à une échelle encore plus grande. En France, ce sont les marais de Bouin et Bourgneuf (en Loire-Atlantique et Vendèe) ; puis plus récemment la région des "Moeres" de la Flandre française. En Belgique, c'est l'ensemble des polders de Veurne. II est trop tôt pour en connaitre les conclusions, mass on en attend beaucoup pour juger de l'importance des surfaces à traiter pour obtenir une persistance des effets. De façon tout empirique, on peut dire que 500 à 1000 ha dans des régions de polders paraissent bien être le minimum convenant à une lutte organisée.

\section{EMPLOI COORDONNE DU PIEGEAGE ET DE L'EMPOISONNEMENT}

Il est impossible à l'heure actuelle d'établir une doctrine nette concernant l'emploi, exclusif ou combiné, du piège et du poison. Il est nécessaire d'attendre pour cela les résultats de toutes les expériences en cours. Quelques remarques peuvent néanmoins être faites dès maintenant.

L'empoisonnement, pas plus que le piègeage, n'est susceptible de constituer le remède miracle permettant de se débarasser du Rat musqué de raçon aisée, définitive et bon marché. Quels que soient les moyens employés, un effort ordonné et soutenu est strictement indispensable.

Cependant les premiers résultats obtenus avec les appâts empoisonnés aissent espérer qu'à condition d'opèrer sur des surfaces importantes, l'empoisonnement permet d'obtenir des résultats durables en n'exigeant que des moyens moindres que ceux nécessités par un piégeage aboutissant au même résultat.

Pour maintenir les résultats obtenus dans les zones traitées au poison, le piègeage apparait mieux adapté et plus sûr que les appâts empoisonnés, lotamment parce qu'il peut permettre de contrôler de façon permanente les principales voies de réinfestation.

Un personnel spécialisè et compétent est aussi indispensable avec l'empoisonnement qu'avec le piègeage pour des raisons d'efficacité et de sécurité. Là où des organisations de piègeurs professionels existent, il apparait indispensable de les maintenir, mais de revoir leur programme d'activité en fonction des possibilités nouvelles offertes par l'empoisonnement. Il est probable qu'une action plus efficace peut etre obtenue sur une zone plus importante avec un même nombre de edestructeurs". 


\section{RISQUES ENTRAINES PAR LES APPATS EMPOISONNES}

A l'égard de l'Homme, et notamment de ceux qui sont chargés de la préparation et de l'épandage des appâts, les anticoagulants ne présentent qu'un risque d'accident pratiquement nul, si les précautions élémentaires et nabituelles sont prises : se laver les mains, ne pas fumer, stocker les produits et préparations à l'abri des autres personnes, etc. Toute garantie sera obtenue si les "destructeurs" sont des personnes bien averties de ce qu'il faut et de se qu'il ne faut pas faire.

Le bétail (bovins, ovins, caprins) est fortement résistant aux anticoagulants. Une consommation accidentelle et non renouvelée, d'appâts empoisonnés n'entraine pas de risque. Ceci ne dispense pas de prendre des précautions et il est prudent de ne traiter les mares, abreuvoirs, fossés... dune prairie quaprès évacuation du bétail et d'enlever les restes d'appâts non consommés avant d'y remettre les animaux.

Le Cochon est un animal très sensible à tous les anticoagularits. Une consommation, même minime, d'appâts entraine un risque certain d'empoisonnement mortel. Il faut éviter absolument que des Cochons puissent manger des appâts ou des cadavres de Rats musqués intoxiqués. Des précautions crès sérieuses devront être prises pour que des restes d'appâts ne puissent ¿̇tre accidentellement mélangès à leur nourriture. (Attention aux instruments ayant servi à la préparation des appâts !).

Le Chien et le Chat sont également très sensibles aux anticoagulants. Mais la nature des appâts utilisés et des lieux traités rendent improbables des accidents. II convient d'éviter que ces animaux ne consomment des cadavres.

Parmi les espèces sauvages, nous distinguons: les Rongeurs aquatiques, le gros Gibier, les Lievres et Lapins, les Oiseaux aquatiques, et le Poisson. Mais soulignons, dès maintenant, que nous ne possédons que peu de renseignements directs à leur sujet. II faut donc étendre à leur cas, les résultats connus sur des animaux voisins d'élevage ou de laboratoire, ce qui en matière de toxicologie comporte toujours un risque d'erreur.

Parmi les Rongeurs aquatiques, le Surmulot et les Campagnols d'eau sont connus comme sensibles aux anticoagulants. Les appâts employés sont de nature à être consommés par eux. "l existe donc un risque certain à leur egard, mais personne ne le regrettera.

Le Cerf et le Chevreuil sont, peut-on penser, à rapprocher des Ruminants domestiques et iusqu'à preuve du contraire, la possibilité d'un accident à leur égard apparait comme très faible. II n'en est évidemment pas de même pour le Sanglier. II est prudent d'estimer que cet animal doit être aussi sensible que le Cochon et là où il fréquente les lieux à Rat musqué, il n'y a guère que l'empoisonnement sur radeau qui puisse apporter des garanties certaines.

Le Lapin domestique est nettement résistant aux anticoagulants. On peut supposer qu'il en est de mème pour le Lapin de Garenne. Quant au Lièvre, sa sensibilité à ces toxiques nous est inconnue et divers exemples connus incitent à penser quil est prudent de le considérer à priori comme plus sensible que le Lapin. Des précautions s'imposent donc à son égard. Là où des Lièvres fréquentent le bord des eaux, il faudra éviter d'épandre des quantités excessives d'appâts susceptibles de laisser des restes importants et il faut même envisager de ne placer les appâts que là où ils seront hors de portée des Lièvres. 
Les oiseaux sont prétendus être très résistants aux anticoagulants. Si on en juge d'après ce que nous savons sur la Poule et autres Gallinacées, c'est exact. Mais il apparait bien que cette affirmation ne peut pas être généralisée à l'ensemble des oiseaux. Notamment nous sommes encore mal informés sur les Canards. Des essais sont prévus cette année à cet effet, mais divers échos et quelques essais préliminaires tendent à montrer que le risque d'accident sur ces oiseaux ne serait pas nul.

En ce qui concerne les Poissons, rien dans la litterature scientifique n'indique que ceux-ci sont sensibles aux anticoagulants. Leur physiologie est très diffèrente de celle des mammifères et, en outre, les produits employés sont insolubles dans l'eau. Si d'autres raisons ne les avaient déjà condamnés, la prudence commanderait d'éviter l'emploi des sels solubles des anticoagulants pour la destruction du Rat musqué.

\section{CONCLUSION}

Comme on le voit, l'emploi des appâts empoisonnés aux anticoagulants ouvre des perspectives intéressantes pour la lutte contre le Rat musqué. Mais bien des points sont encore à préciser ainsi qu'on l'a vu à chaque paragraphe de cette note. Les applications conservent donc encore un caractère expérimental certain.

D'autre part, il faut se garder d'un engouement excessif et surtout d'utilisations inconsidérées ne respectant pas les précautions que la technique et la sécurité imposent. S'il n'en était pas ainsi, on risquerait d'entrainer le discrédit, voire l'interdiction, d'une méthode qui, judicieusement employée, serait d'un grand secours dans la lutte contre le Rat musqué.

$$
\text { Jouy-en-Josas, le } 25 \text { mars } 1970 .
$$

